www.nature.com/hr

\title{
The effect of treatment on monocyte and lymphocyte cytokine release in patients with aldosteronoma
}

\author{
Hypertension Research (2012) 35, 123-125; doi:10.1038/hr.2011.142; published online 25 August 2011
}

Primary aldosteronism is one of the leading causes of secondary hypertension. It may be responsible for as many as $5-13 \%$ of all cases of arterial hypertension and $\sim 20 \%$ cases of treatment-resistant hypertension. ${ }^{1}$ Although mineralocorticoid receptor antagonists, such as spironolactone and eplerenone, are considered an alternative to adrenalectomy for patients with contraindications to surgery, for patients who decline surgery and for patients with bilateral adrenal hyperplasia, conflicting evidence exists on whether these agents are as effective as surgical treatment. ${ }^{1}$ In our study, we compared the effects of adrenalectomy and pharmacological treatment with mineralocorticoid receptor antagonists on monocyte and lymphocyte cytokine release in patients with aldosteronoma. Of the many monocyte- and lymphocyte-derived factors, we elected to measure the levels of tumor necrosis factor- $\alpha$ (TNF- $\alpha$ ), interleukin- $1 \beta$, interleukin-6, interferon- $\gamma$ and interleukin-2. These cytokines produce a multi-directional proatherogenic effect, ${ }^{2-5}$ and our team has long-term experience with their assessment. ${ }^{6,7}$ This study was approved by a local ethical committee.

Our study included four female patients (24-38 years old) who had aldosteroneproducing adrenocortical adenomas and were admitted to our department because of grade 3 arterial hypertension resistant to standard hypotensive agents. These subjects had not been treated previously with mineralocorticoid receptor antagonists or other agents that may markedly affect the aldosterone -to-renin ratio, and other antihypertensive drugs (with the exception of verapamil and prazosin) had been discontinued at least 2 weeks before hospitalization. In all four patients, supine plasma renin activity was suppressed, whereas supine plasma aldosterone levels were elevated, which resulted in elevated aldosterone/renin ratios (Table 1). The plasma renin activity and the plasma aldosterone levels remained at the same levels or increased only slightly after standing for 4 hours. Provocative tests, including the saline infusion test $(21$ during 4 hours) and the fludrocortisone suppression test, revealed unsuppressed plasma aldosterone levels (Table 1). In each patient, a computed tomography scan of the abdomen revealed a well-circumscribed, non-enhancing

Table 1 Baseline characteristics of patients ${ }^{a}$

\begin{tabular}{|c|c|c|c|c|c|c|}
\hline & \multicolumn{4}{|c|}{ Patients with aldosteronoma } & \multirow{2}{*}{$\begin{array}{c}\text { Patients with } \\
\text { essential } \\
\text { hypertension } \\
(\mathrm{n}=10)^{\mathrm{b}}\end{array}$} & \multirow{2}{*}{$\begin{array}{c}\text { Apparently } \\
\text { healthy } \\
\text { women } \\
(\mathrm{n}=12)^{\mathrm{b}}\end{array}$} \\
\hline & Patient 1 & Patient 2 & Patient 3 & Patient 4 & & \\
\hline Age (years) & 37 & 34 & 29 & 31 & $32(4)$ & $32(5)$ \\
\hline Body mass index $\left(\mathrm{kg} \mathrm{m}^{-2}\right)$ & 23.2 & 25.1 & 25.5 & 24.1 & $24.2(2.3)$ & $23.8(1.5)$ \\
\hline Systolic blood pressure $(\mathrm{mm} \mathrm{Hg})^{\mathrm{c}}$ & 192 & 194 & 198 & 187 & $193(8)$ & $119(7)$ \\
\hline Supine plasma renin activity $\left(\mu \mathrm{g} \mathrm{I}^{-1} \mathrm{~h}^{-1} ; 0.3-2.8\right)^{\mathrm{d}}$ & 0.02 & 0.02 & 0.01 & 0.04 & $1.5(1.0)$ & $1.2(0.5)$ \\
\hline Supine plasma aldosterone levels $\left(\mathrm{pmol}^{-1} ; 80-420\right)^{\mathrm{d}}$ & 1816 & 1728 & 1997 & 1331 & $260(85)$ & $164(29)$ \\
\hline Aldosterone/renin ratio $\left(\mathrm{pmol}^{-1}\right) /\left(\mu \mathrm{g} \mathrm{I}^{-1} \mathrm{~h}^{-1}\right)$ & 90800 & 86400 & 199700 & 33275 & $173(60)$ & $137(48)$ \\
\hline $\begin{array}{l}\text { Plasma aldosterone levels in the saline infusion } \\
\text { test }\left(\mathrm{pmol} \mathrm{I}^{-1}\right)(<139)^{\mathrm{d}}\end{array}$ & 1690 & 1345 & 1720 & 901 & Not investigated & Not investigated \\
\hline $\begin{array}{l}\text { Plasma aldosterone levels in the fludrocortisone } \\
\left.\text { suppression test (pmol } \mathrm{I}^{-1} ;<166\right)^{\mathrm{d}}\end{array}$ & 1123 & 1475 & 1460 & 985 & Not investigated & Not investigated \\
\hline
\end{tabular}

aExclusion criteria: any acute and chronic inflammatory processes; any autoimmune disorders; unstable coronary artery disease, myocardial infarction or stroke within 6 months preceding the study; $\mathrm{BMI}>40 \mathrm{~kg} \mathrm{~m}^{-2}$; impaired renal or hepatic function; malabsorption syndromes; pregnancy or lactation; previous treatment with mineralocorticoid receptor antagonists; concomitant treatment with other drugs known to interact with mineralocorticoid receptor antagonists and poor patient compliance.

bThe mean (s.d.).

cThe average of three readings obtained at 5-min intervals.

dNormal limits. 
or slightly enhancing unilateral hypodense mass $(12-20 \mathrm{~mm}$ in diameter) in the left (patients 1 and 4) or the right (patients 2 and 3 ) adrenal gland, suggestive of a benign adenoma. Because of the young age of the patients (below 40 years) and the presence of a tumor $(>1 \mathrm{~cm}),{ }^{1}$ adrenal vein sampling was not performed. All subjects started treatment with spironolactone, but because of the side effects, this agent was replaced with eplerenone in patient 2. After 2 months, patients 1 and 4 underwent laparoscopic adrenalectomy. The remaining two patients (patients 2 and 3), who declined surgical intervention, continued treatment with spironolactone or eplerenone during the entire observation period (24 months). Laparoscopic adrenalectomy resulted in a normalization of blood pressure, and therefore, after surgery, the two patients who underwent surgery did not receive any pharmacological treatment. Normal blood pressure was also achieved in subjects treated with mineralocorticoid receptor antagonists (patient 3 also received hydrochlorothiazide). Patients with primary aldosteronism were compared with 10 age-, sex- and weightmatched individuals with poorly controlled grade 3 essential hypertension and 12 apparently healthy women. Essential hypertensive patients had not previously received any mineralocorticoid receptor antagonist and were not treated with these agents during the entire observation period. After modification of the therapy, normalization of blood pressure was achieved within 2 months. Cultures of lipopolysaccharide-stimulated monocytes and phytohemagglutinin-stimulated Tcells were performed in triplicate as described previously. ${ }^{6,7}$ Monocyte release of TNF- $\alpha$, interleukin- $1 \beta$ and interleukin-6 and lymphocyte release of TNF- $\alpha$, interferon- $\gamma$ and interleukin-2 were estimated using the commercial enzyme-linked immunosorbent assay kits (R\&D Systems, McKinley Place
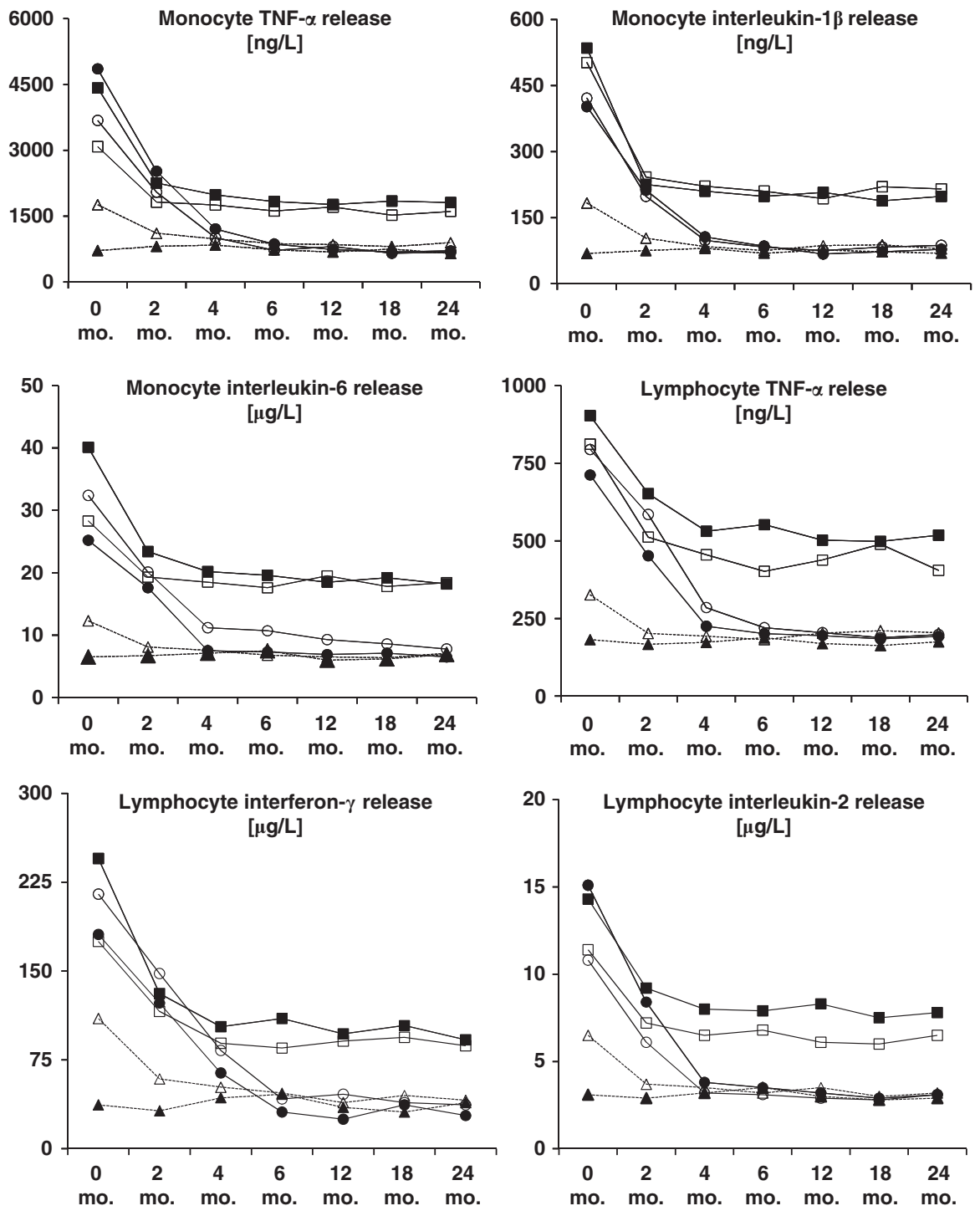

Figure 1 The effect of treatment on monocyte and lymphocyte cytokine release in patients with primary aldosteronism. Patient 1 (open circles) and patient four (closed circles) were initially treated with spironolactone and, two months later, underwent unilateral adrenalectomy. Patient 2 (open squares) was initially treated with spironolactone but starting in month 4, received eplerenone (200 mg daily). Patient 3 (closed squares) received spironolactone (100 mg daily) during the whole observation period (24 months). Patients with poorly controlled essential hypertension (open triangles) achieved normalization of blood pressure within 2 months of intensive antihypertensive therapy. Apparently healthy subjects (closed triangles) did not receive any treatment. For patients with poorly controlled essential hypertension and apparently healthy subjects, the mean cytokine release in each time point is shown. 
N.E. Minneapolis, MN, USA) according to the manufacturer's instructions.

Compared with patients with poorly controlled essential hypertension, patients with primary aldosteronism showed increased monocyte release of TNF- $\alpha(4010 \pm 831 v s$. $1760 \pm 305 \mathrm{ngl}^{-1}$; healthy subjects: $720 \pm$ $\left.155 \mathrm{ngl}^{-1}\right)$, interleukin-1 $\beta \quad(465 \pm 13 \quad$ vs. $183 \pm 14 \mathrm{ngl}^{-1}$; healthy subjects: $68 \pm$ $\left.8 \mathrm{ng}^{-1}\right)$ and interleukin-6 (31.5 \pm 5.1 vs. $12.3 \pm 2.0 \mu \mathrm{gl}^{-1}$; healthy subjects: $6.5 \pm$ $\left.1.2 \mu \mathrm{gl}^{-1}\right)$ and increased lymphocyte release of TNF- $\alpha \quad\left(805 \pm 58\right.$ vs. $326 \pm 22 \mathrm{ngl}^{-1}$; healthy subjects: $\left.182 \pm 19 \mathrm{ngl}^{-1}\right)$, interferon$\gamma\left(204 \pm 24\right.$ vs. $110 \pm 14 \mu \mathrm{gl}^{-1}$; healthy subjects: $\left.\quad 37 \pm 5 \mu \mathrm{gl}^{-1}\right)$ and interleukin-2 $\left(12.9 \pm 3.0 \quad\right.$ vs. $\quad 6.5 \pm 1.2 \mu \mathrm{gl}^{-1}$; healthy subjects: $\left.3.1 \pm 0.6 \mu \mathrm{g} \mathrm{l}^{-1}\right)$. Monocyte and lymphocyte cytokine release was higher in patients with essential hypertension than in subjects with normal blood pressure. Baseline monocyte and lymphocyte cytokine release showed a weak correlation with systolic and diastolic blood pressures ( $r$ values between 0.40 and $0.49, P<0.01)$ and with plasma aldosterone levels $(r$ values between 0.42 and $0.53, P<0.01)$ but did not depend on the type of antihypertensive medication the patients were taking. Adrenalectomy and mineralocorticoid receptor antagonists resulted in a decrease in monocyte and lymphocyte cytokine release in all patients studied (Figure 1). In the two subjects who underwent adrenalectomy, post-surgery cytokine release did not differ compared with that in apparently healthy subjects. In the remaining two patents, who continued pharmacological treatment with spironolactone and eplerenone, only a partial reduction in monocyte and lymphocyte cytokine release was observed, and at the end of observation period, the cytokine release in these subjects exceeded that observed in the apparently healthy subjects.
To the best of our knowledge, this study is the first to show that the pattern of abnormalities in monocyte and lymphocyte cytokine secretion differs between patients with primary aldosteronism and matched subjects with essential hypertension. Significant differences between these groups with respect to cytokine release are in part a consequence of differences in plasma aldosterone levels. In patients with aldosteronoma, the hypertension-induced increase in cytokine secretion is probably potentiated by the additive effect of excess aldosterone. Taking into account that the high levels of the cytokines that were measured in our patients are associated with increased short-term and longterm cardiovascular risk $^{2-5}$ and the pivotal role of monocytes and lymphocytes in atherogenesis, ${ }^{4,5}$ the enhanced secretory function of these cells may make subjects with aldosteronoma more prone to the earlier development and faster progression of atherosclerosis compared with essential hypertensive patients. Both pharmacological and surgical treatments reduced cytokine release, but only the latter treatment option decreased cytokine release to the level observed in apparently healthy subjects. Although mineralocorticoid receptor antagonists produced multi-directional anti-inflammatory effects, their monocyte- and lymphocyte-suppressive effects did not increase with time, and cytokine release was higher than in control subjects during the entire observation period. These findings are in disagreement with those of other authors ${ }^{8-10}$ who observed similar cardiovascular and nephroprotective benefits after adrenalectomy and spironolactone treatment. However, their study populations included both patients with aldosteronoma (most of whom underwent adrenalectomy) and patients with bilateral adrenal hyperplasia (treated with spironolactone), and this fact may explain the differences between our results and their results. In our opinion, patients with aldosteronoma experience more benefits when treated with surgery, even if mineralocorticoid receptor antagonists effectively reduce blood pressure.

\section{Robert Krysiak and Boguslaw Okopien}

Department of Internal Medicine and Clinical Pharmacology, Medical University of Silesia, Katowice, Poland E-mail: r.krysiak@interia.pl

1 Young W. Primary aldosteronism: renaissance of the syndrome. Clin Endocrinol 2007; 66: 607-618.

2 Hansson GK. Inflammatory mechanisms in atherosclerosis. J Thromb Hemost 2009; 7(Suppl 1): 328-331.

3 Tedgui A, Mallat Z. Cytokines in atherosclerosis: pathogenic and regulatory pathways. Physiol Rev 2006; 86: 515-581.

4 Kher N, Marsh JD. Pathobiology of atherosclerosis - a brief review. Semin Thromb Hemost 2004; 30: 665-672.

5 Schroecksnadel K, Frick B, Winkler C, Fuchs D. Crucial role of interferon-gamma and stimulated macrophages in cardiovascular disease. Curr Vasc Pharmacol 2006; 4: 205-213.

6 Okopieñ B, Krysiak R, Kowalski J, Madej A, Belowski D, Zielinski M, Herman ZS. Monocyte release of tumor necrosis factor-alpha and interleukin-1beta in primary type Ila and IIb dyslipidemic patients treated with statins or fibrates. J Cardiovasc Pharmacol 2005; 46: 377-386.

7 Okopieñ B, Krysiak R, Kowalski J, Madej A, Belowski D, Zielinski M, Labuzek K, Herman ZS. The effect of statins and fibrates on interferon-gamma and interleukin-2 release in patients with primary type II dyslipidemia. Atherosclerosis 2004; 176: 327-335.

8 Catena C, Colussi G, Lapenna R, Nadalini E, Chiuch A, Gianfagna P, Sechi LA. Long-term cardiac effects of adrenalectomy or mineralocorticoid antagonists in patients with primary aldosteronism. Hypertension 2007; 50: 911-918.

9 Catena C, Colussi G, Nadalini E, Chiuch A, Baroselli S, Lapenna R, Sechi LA. Cardiovascular outcomes in patients with primary aldosteronism after treatment. Arch Intern Med 2008; 168: 80-85.

10 Sechi LA, Novello M, Lapenna R, Baroselli S, Nadalini E, Colussi GL, Catena C. Long-term renal outcomes in patients with primary aldosteronism. JAMA 2006; 295: 2638-2645. 